\title{
Live surgery
}

S ome plastic surgery seminars highlight live surgery, during which surgery is performed on a closed circuit television linked between an audience of surgeons and a nearby operating room. The surgeons who have been chosen to operate are high profile innovators with extensive experience. Their demonstration of surgery is of great value to patients and surgeons alike, especially when uncommonly performed variations can be distilled and simplified. By this, a surgeon's learning curve is vaulted far ahead of what could be achieved any other way except by visiting the surgeon. It takes the error out of trial and error.

Lately though, some surgeons have been saying, "why not just have videos?" This is a good question.

To arrange a live surgical demonstration, you need a reliable patient who is willing to have surgery at low or no cost. You need a well-known surgeon who is willing to demonstrate a difficult operation, a facility, an audiovisual department and a moderator. To plan live surgery you need to consider the following:

First, surgeons of this calibre are booked several years in advance. The contact is usually personal and made face-toface at international meetings. Visiting surgeons come as a favour to someone they know and for an interesting learning experience. They seldom receive an honorarium but become honourary members of the society which invites them. The inviting society pays the expenses.

Second, the host surgeon needs to select a patient from his or her own practice who has a problem appropriate to the operation to be performed. The patient will be discussed by telephone with the visiting surgeon, and photographs sent. The day before surgery, the visiting surgeon will assess the patient, adding information to the host surgeon's consultation if needed. If the initial patient declines surgery at the last moment, it is advisable to have a backup patient ready, so that the live surgical demonstration can proceed as planned. To be fair, the host surgeon should later operate on the second patient under the same arrangement to avoid disappointment if the first patient is chosen.

Third, the audiovisual arrangement has to be professionally done. The operating room has to be big enough to accommodate cameras, trolleys, electrical connections to the auditorium and camera personnel. Some operations can be very difficult to photograph (eg, rhinoplasties) and to show the dark recesses which are the very parts of the operation the viewer usually wants to see. Also, televising a procedure requires that the surgeon works more slowly than usual, and a surgical day may go on longer than predicted. Then you run into overtime, audiences drifting away and late recovery rooms. It is sometimes better to under-book.

Fourth, the modera-

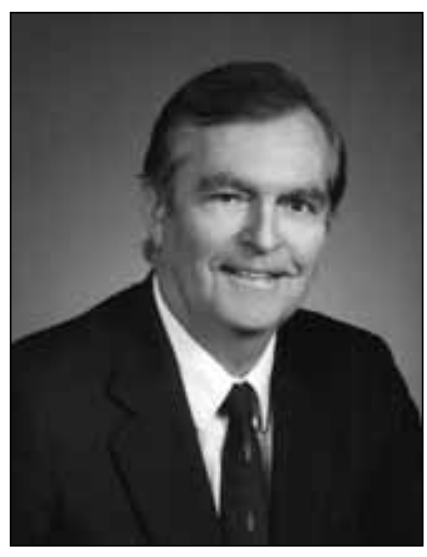

John R Taylor tor has to be a surgeon who knows the operating surgeon well. He or she has to know when to interrupt the surgeon with questions from the audience. This usually occurs when something routine is going on.

Fifth, you may need to run two operating rooms simultaneously, for two reasons. There are routine parts of an operation, such as suturing, which create a lull, and surgery is often bilateral. Watching the second side can clarify a point, but often all the viewer needs to see is one side. During the lull, the moderator can switch to the second room, or interrupt one operating room when the other surgeon wants to illustrate a particularly important point.

Sixth, it should be possible to run one operating room only, and intersperse the down times with prerecorded material as long as it is well edited and of a short duration.

Live surgical demonstrations will always be attractive to surgeons in practice. It is a direct way of comparing our techniques with those of our well-known colleague leaders, particularly in the less commonly performed operations and when there are multiple choices where no one approach seems obviously superior. Live surgery gives an immediacy not found in edited surgery and gives the audience access to ask questions not afforded by any other medium.

It is expensive and difficult to arrange, but well worth the effort.

John R Taylor 